\title{
ARTICLE OPEN Dielectric properties of hexagonal boron nitride and transition metal dichalcogenides: from monolayer to bulk
}

\author{
Akash Laturia ${ }^{1}$, Maarten L. Van de Put ${ }^{1}{ }^{1}$ and William G. Vandenberghe ${ }^{1}$
}

\begin{abstract}
Hexagonal boron nitride (h-BN) and semiconducting transition metal dichalcogenides (TMDs) promise greatly improved electrostatic control in future scaled electronic devices. To quantify the prospects of these materials in devices, we calculate the outof-plane and in-plane dielectric constant from first principles for TMDs in trigonal prismatic and octahedral coordination, as well as for $\mathrm{h}-\mathrm{BN}$, with a thickness ranging from monolayer and bilayer to bulk. Both the ionic and electronic contribution to the dielectric response are computed. Our calculations show that the out-of-plane dielectric response for the transition-metal dichalcogenides is dominated by its electronic component and that the dielectric constant increases with increasing chalcogen atomic number. Overall, the out-of-plane dielectric constant of the TMDs and h-BN increases by less than 15\% as the number of layers is increased from monolayer to bulk, while the in-plane component remains unchanged. Our computations also reveal that for octahedrally coordinated TMDs the ionic (static) contribution to the dielectric response is very high (4.5 times the electronic contribution) in the in-plane direction. This indicates that semiconducting TMDs in the tetragonal phase will suffer from excessive polar-optical scattering thereby deteriorating their electronic transport properties.
\end{abstract}

npj 2D Materials and Applications (2018)2:6 ; doi:10.1038/s41699-018-0050-x

\section{INTRODUCTION}

The continued miniaturization of silicon-based electronics along with the exfoliation of graphene from graphite has motivated extensive research toward layered two-dimensional (2D) materials. However, since graphene does not have a band-gap, it is not well suited for digital electronics applications. ${ }^{1}$ Consequently, significant research effort has been directed toward 2D semiconductors. Indeed several classes of 2D semiconductors like boron-nitride (h$\mathrm{BN})$, transition metal dichalcogenides (TMDs), silicene, phosphorene, layered transition metal oxides, and hydroxides have been studied in the recent past. ${ }^{2-5}$

TMDs are layered materials with chemical formula $M X_{2}$ where $M$ is a transition metal and $X$ is a chalcogen. Many different TMDs are possible because of the various permutations of the transition metal and the chalcogen that can be made. TMDs are either metallic, semi-metallic, semiconductors, topological insulators, or superconductors. ${ }^{6-8}$ As most of the semiconducting TMDs have a bandgap $92 \mathrm{eV}$, they are great candidates for future electronic devices. ${ }^{10}$ In addition, their atomically thin nature enables further miniaturization of electronic devices. Also, many monolayer TMDs have a direct bandgap, enabling their use in the field of optoelectronics. ${ }^{11}$ Structurally, TMDs have strong in-plane covalent bonding and weak out-of-plane van der Waals bonding. Just like in graphene, the weak van der Waals force between the layers facilitates exfoliation of individual or multiple layers. The versatility of the electronic and optical properties of layered materials (TMDs) and the ability to exfoliate monolayers and few layers has propelled extensive research in the field of TMDs. ${ }^{12-15}$

Another intriguing 2D material with a manifold of suggested applications is hexagonal boron-nitride (h-BN). It is a direct bandgap material with a large gap ${ }^{16}$ of $5.97 \mathrm{eV}$. Therefore it is being investigated as an alternative to gallium nitride (GaN) for ultraviolet lasing applications, such as optical storage and photocatalysis. ${ }^{16}$ Also, $\mathrm{h}-\mathrm{BN}$ is a potential gate dielectric for TMD-based field-effect transistors (FETs). The 2D nature of h-BN, along with the absence of trapped charges or dangling bonds, yields improved performance when integrated with molybdenum disulphide $\mathrm{MoS}_{2}$ and other TMDs compared to TMDs on a silicon dioxide $\left(\mathrm{SiO}_{2}\right)$ substrate. ${ }^{17-19}$

The great potential of $2 \mathrm{D}$ materials, mainly TMDs and h-BN, for future electronic devices requires a detailed study of their electronic transport properties. One of the key properties in this regard is the dielectric response of these materials. Knowledge of the dielectric response is critical to estimate both their electrostatic properties, as well as polar electronic scattering processes. Due to the different nature of bonds in the in-plane and the outof-plane direction, the dielectric response of 2D materials is anisotropic. Most of the previous studies have either focused only on the in-plane dielectric constant or have done the analysis of dielectric properties for a specific 2D material. ${ }^{20-23}$ An extensive study of the in-plane and the out-of-plane electrostatic dielectric response is missing, however.

Earlier work on the dielectric constant of $\mathrm{MoS}_{2}$ demonstrated the influence of the surrounding media on the dielectric constant. $^{24}$ In particular, the out-of-plane dielectric constant (relative permittivity) of the 2D crystal was defined as an average of the dielectric constant of the surrounding media. ${ }^{25}$ But this is not a viable approach as it does not account for the polarization in the TMD. Thus, in order to accurately simulate the electrostatic behavior and the effect of polar-optical electron-phonon scattering on mobility degradation, it is necessary to compute the intrinsic dielectric response of the $2 \mathrm{D}$ materials.

${ }^{1}$ Department of Materials Science and Engineering, The University of Texas at Dallas, Richardson, TX, USA

Correspondence: William G. Vandenberghe (wxv101020@utdallas.edu)

Received: 3 October 2017 Revised: 26 January 2018 Accepted: 1 February 2018

Published online: 08 March 2018 
Despite the importance of dielectric properties, its study has received less attention. A few of the studies related to the dielectric constant include a first principles calculation of the outof-plane dielectric constant of $\mathrm{MoS}_{2}$ and h-BN. ${ }^{26,27}$ However, an exhaustive study of both the in-plane and out-of-plane dielectric properties of the other $2 \mathrm{D}$ materials is missing.

In this paper, we calculate the macroscopic optical and static relative permittivity values for free-standing monolayer, bilayer and bulk TMDs corresponding to the in-plane and out-of-plane direction. We consider semiconducting TMDs: molybdenum (Mo), tungsten $(\mathrm{W})$, zirconium $(\mathrm{Zr})$, and hafnium $(\mathrm{Hf})$ based TMDs and $\mathrm{h}$ $\mathrm{BN}$. We find that the in-plane dielectric constant is larger than the out-of-plane dielectric response for all the 2D materials. We also find that the electronic component dominates the dielectric response except for TMDs in the tetragonal phase where the ionic contribution is dominant in the in-plane direction. Finally, we also analyze the variation of optical and static dielectric constant (relative permittivity) of h-BN as function of 1-5 layers and how monolayer and bilayer values for TMDs compare against their respective bulk values. Our calculations show that the in-plane dielectric constant shows negligible thickness dependence for both TMDs in the hexagonal phase and h-BN.

This paper is organized as follows. Firstly, we outline and discuss the results obatined from the ab initio calculations of the macroscopic dielectric response of the 2D materials. Following this, we give information about the parameters used in the $a b$ initio calculations in the Methods section. The methods section also contains the details of the first principles calculation of the electronic and ionic contribution to the dielectric constant. The

Table 1. h-BN: Macroscopic dielectric constant variation with the number of layers

\begin{tabular}{lllll}
\hline Number of layers & $\epsilon_{\|, \infty}$ & $\epsilon_{\|, 0}$ & $\epsilon_{\perp, \infty}$ & $\epsilon_{\perp, 0}$ \\
\hline 1 & 4.97 & 6.82 & 2.89 & 3.29 \\
2 & 4.97 & 6.86 & 2.91 & 3.44 \\
3 & 4.97 & 6.86 & 2.96 & 3.52 \\
4 & 4.96 & 6.86 & 2.97 & 3.58 \\
5 & 4.96 & 6.86 & 2.98 & 3.62 \\
Bulk & 4.98 & 6.93 & 3.03 & 3.76 \\
\hline
\end{tabular}

h-BN: In-plane and out-of-plane dielectric constant for different number of layers. $\epsilon_{\|}$is the in-plane and $\epsilon_{\perp}$ is the out-of-plane dielectric constant. $\epsilon_{0}$ denotes the static dielectric constant; whereas, $\epsilon_{\infty}$ denotes the dielectric constant at optical frequency obtained dielectric constant values need to be normalized corresponding to the thickness of the $2 \mathrm{D}$ materials. Finally, the normalization of the obtained macroscopic dielectric constants of TMDs and h-BN is detailed.

\section{RESULTS AND DISCUSSION}

We have calculated the macroscopic out-of-plane and in-plane dielectric constants for several TMDs and h-BN in monolayer, bilayer and bulk configurations from first principles calculations. In the case of mono- and bi-layers, special care has been taken to isolate its dielectric constant from the environment. More details on the exact procedure can be found in the Methods section. The calculated dielectric constant values for h-BN and TMDs are tabulated in Table 1 and Table 2, respectively. The dielectric constant of h-BN as a function of the number of layers is shown in Fig. 1. Both the in-plane and out-of-plane dielectric constant for TMDs (from Table 2) are visually represented in Fig. 2 and Fig. 3, respectively. In these figures, $\epsilon_{0}$ represents the macroscopic static response containing both the ionic and the electronic response; whereas, $\epsilon_{\infty}$ represents only the electronic component (which is also the macroscopic optical dielectric constant).

Inspecting the dielectric constant values in Tables 1-2, we observe that the dielectric constant is always higher in the inplane direction than in the out-of-plane direction. Indeed, the outof-plane interlayer interaction being a van der Waals interaction will be less polarizable compared to the in-plane covalent bonding.

Figure 1 shows that the dielectric constant of h-BN in the out-ofplane direction ranges from 3.29 to 3.76 , and shows thickness dependence. The in-plane dielectric constant on the other hand is relatively constant, ranging only from 6.82 to 6.93 , as the number of layers increases from monolayer to bulk. Our findings are in contrast with the findings in ref. ${ }^{26}$, which found a strong layerdependence for $\mathrm{h}-\mathrm{BN}$, and ref. ${ }^{20}$, where a dielectric constant of unity was obtained. The discrepancy between our current and those previous findings stems from the different definition of thickness employed. The definition we use, computing the $n$-layer thickness from the structure of an $n+1$-layer structure, aligns more closely to the thickness values which would be found in a multilayer 2D material stack and will generally be more relevant. We have reported the obtained material thickness in Table 3. One of the important aspects of these calculations is that these dielectric constant values can be renormalized for different values of thickness obtained in heterostructures.

The two important equations which govern the rescaling of the in-plane $\left(\epsilon_{2 \mathrm{D}, \|}\right)$ and out-of-plane dielectric constant $\left(\epsilon_{2 \mathrm{D}, \perp}\right)$ with

\begin{tabular}{|c|c|c|c|c|c|c|c|c|c|c|c|c|}
\hline Material & $\perp$ & $\|$ & $\perp$ & $\|$ & $\perp$ & $\|$ & $\perp$ & $\|$ & $\perp$ & $\|$ & $\perp$ & $\|$ \\
\hline $\mathrm{MoS}_{2}(\mathrm{H})$ & 6.1 & 15.4 & 6.2 & 15.5 & 6.5 & 15.6 & 6.6 & 15.7 & 6.9 & 15.8 & 6.9 & 15.9 \\
\hline $\mathrm{MoSe}_{2}(\mathrm{H})$ & 7.2 & 16.8 & 7.2 & 17.4 & 7.6 & 17.0 & 7.7 & 17.5 & 8.1 & 17.2 & 8.5 & 17.7 \\
\hline $\mathrm{MoTe}_{2}(\mathrm{H})$ & 8.9 & 19.7 & 9.1 & 21.6 & 9.4 & 19.9 & 9.8 & 21.7 & 10.1 & 20.1 & 10.4 & 21.9 \\
\hline $\mathrm{WSe}_{2}(\mathrm{H})$ & 7.4 & 15.3 & 7.4 & 15.6 & 7.5 & 15.5 & 7.6 & 15.7 & 7.7 & 15.6 & 7.8 & 15.9 \\
\hline $\mathrm{HfS}_{2}(\mathrm{~T})$ & 5.6 & 10.2 & 6.6 & 53.6 & 5.6 & 10.2 & 6.3 & 51.3 & 5.8 & 10.4 & 6.5 & 48.9 \\
\hline $\mathrm{HfSe}_{2}(\mathrm{~T})$ & 6.7 & 13.9 & 7.6 & 83.0 & 6.9 & 13.9 & 7.7 & 77.0 & 7.2 & 13.9 & 7.8 & 71.2 \\
\hline $\mathrm{ZrS}_{2}(\mathrm{~T})$ & 5.7 & 11.2 & 6.8 & 66.7 & 5.9 & 11.3 & 6.9 & 61.3 & 6.2 & 11.4 & 7.0 & 65.3 \\
\hline
\end{tabular}



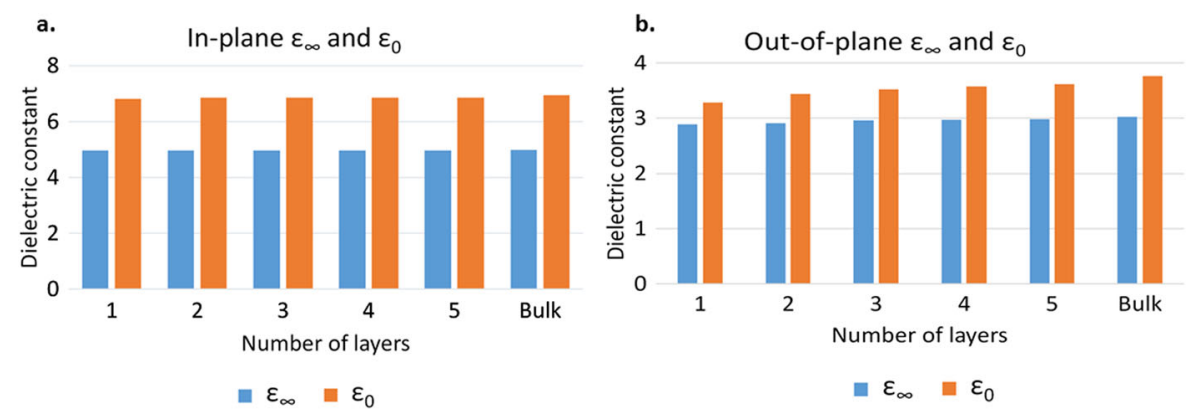

Fig. 1 h-BN: Layer dependent macroscopic dielectric constant. a h-BN: In-plane macroscopic dielectric constant. b h-BN: out-of-plane macroscopic dielectric constant. $\epsilon_{\infty}$ represents the macroscopic optical dielectric constant and $\epsilon_{0}$ represents the macroscopic static dielectric constant. The static out-of-plane dielectric constant varies from 3.29 (monolayer) to 3.76 (bulk). The in-plane static dielectric constant varies negligibly from 6.82 (monolayer) to 6.93 (bulk)
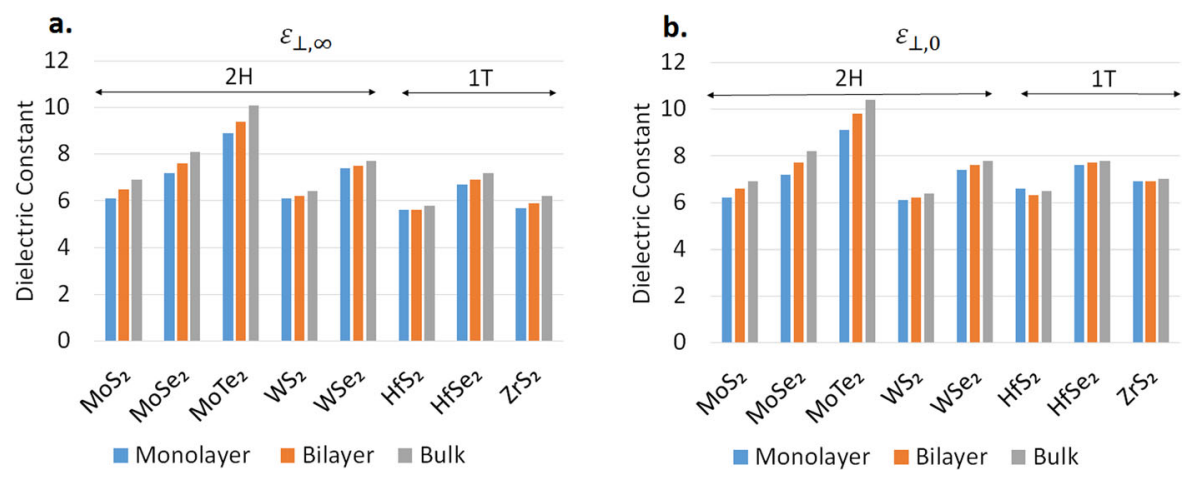

Fig. 2 Out-of-plane macroscopic dielectric constant of monolayer, bilayer and bulk TMDs with 2H and 1T polytype structure. a TMD: out-ofplane macroscopic optical dielectric constant. b TMD: out-of-plane macroscopic static dielectric constant. $\epsilon_{\perp, \infty}$ represents the out-of-plane macroscopic optical dielectric constant and $\epsilon_{\perp, 0}$ represents the macroscopic static out-of-plane dielectric constant. The dielectric constant of $2 \mathrm{H}$ TMDs increase monotonically from monolayer to bulk; whereas, 1T TMDs vary insignificantly with thickness
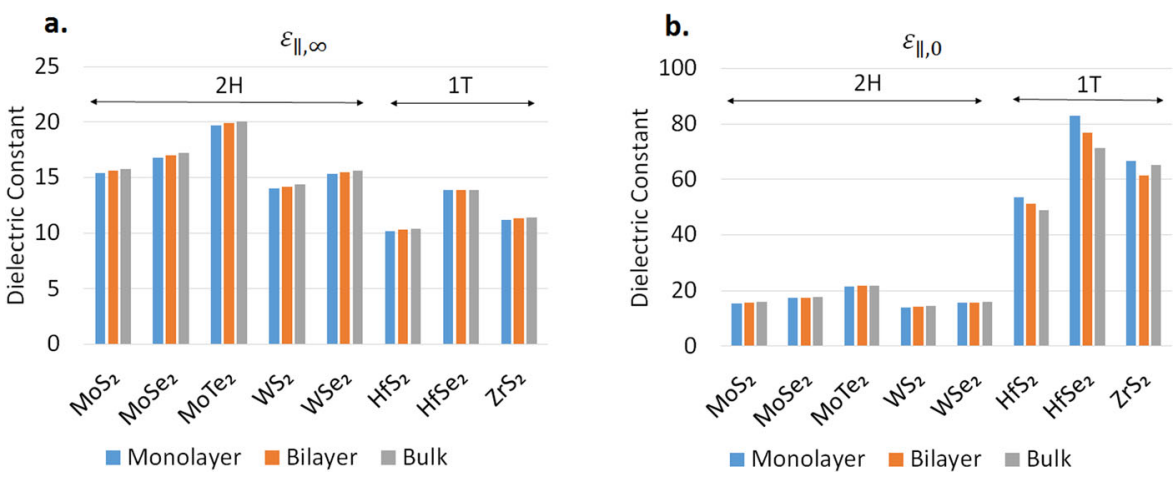

Fig. 3 In-plane macroscopic dielectric constant of monolayer, bilayer and bulk TMDs with $2 \mathrm{H}$ and 1T polytype structure. a TMD: in-plane macroscopic optical dielectric constant. b TMD: in-plane macroscopic static dielectric constant. $\epsilon_{\|, \infty}$ represents the in-plane macroscopic optical dielectric constant and $\epsilon_{\| .0}$ represents the static macroscopic in-plane dielectric constant. 1T TMDs show a dominant ionic response in the in-plane direction, which is $\sim 4.5$ times the electronic response

thickness are described below where $c$ is the thickness of the supercell containing the $2 \mathrm{D}$ material and $t$ is its thickness.

$\epsilon_{2 \mathrm{D}, \perp}=\left[1+\frac{c}{t}\left(\frac{1}{\epsilon_{\mathrm{SC}, \perp}}-1\right)\right]^{-1}$.

$\epsilon_{2 \mathrm{D}, \|}=1+\frac{c}{t}\left(\epsilon_{\mathrm{SC}, \|}-1\right)$.

In Eq. (1) and Eq. (2), $\epsilon_{\mathrm{SC}, \|}$ and $\epsilon_{\mathrm{SC}, \perp}$ represent the in-plane and the out-of-plane dielectric constant of the supercell. One of the key points to note is that these reported macroscopic dielectric constants $\left(\epsilon_{2 \mathrm{D}, \perp / \|}\right)$ are intrinsic to the $2 \mathrm{D}$ material. If however, we take the perspective of capacitive or optical measurements on a
$2 \mathrm{D}$ material stack, the experimental thickness might be different from the one reported in this manuscript. In this case, Eq. (1) and Eq. (2) can be used to rescale the dielectric constant to a different thickness value by taking $c$ as the thickness from Table $3, \epsilon_{\mathrm{SC}}$ as the dielectric constant from Tables 1 or 2 and $t$ as the experimental thickness. This thickness sensitivity of the 2D dielectric constant is shown in Supplementary Information, where results for free-standing monolayer $\mathrm{MoTe}_{2}$ are depicted. This analysis shows that the macroscopic dielectric tensor of monolayer $\mathrm{MoTe}_{2}$ approaches that of vacuum when an infinite interlayer separation is considered. These results are similar to those calculated for monolayer $\mathrm{MoS}_{2}$ and are expected when dielectric constant of a free-standing monolayer, surrounded by 
Table 3. Simulation parameters used for the calculation of macroscopic dielectric constants of 2D materials

\begin{tabular}{lllll}
\hline Material & $a(\AA)$ & $1 \mathrm{~L}[t(\AA)]$ & $2 \mathrm{~L}[t(\AA)]$ & Bulk $\left[\mathrm{d}_{i}\right]$ \\
\hline $\operatorname{MoS}_{2}(2 \mathrm{H})$ & 3.20 & $6.11(-0.33 \%)$ & $12.21(-0.16 \%)$ & 6.09 \\
$\operatorname{MoSe}_{2}(2 \mathrm{H})$ & 3.35 & $6.49(-1.15 \%)$ & $12.98(-0.15 \%)$ & 6.48 \\
$\operatorname{MoTe}_{2}(2 \mathrm{H})$ & 3.59 & $7.1(-0.28 \%)$ & $14.19(-0.14 \%)$ & 7.08 \\
$\mathrm{WS}_{2}(2 \mathrm{H})$ & 3.21 & $6.12(-0.33 \%)$ & $12.23(-0.16 \%)$ & 6.1 \\
$\mathrm{WSe}_{2}(2 \mathrm{H})$ & 3.33 & $6.48(-0.15 \%)$ & $12.95(-0.15 \%)$ & 6.47 \\
$\mathrm{~h}-\mathrm{BN}_{(\mathrm{H})}$ & 2.51 & $3.17(-1.42 \%)$ & $6.31(-0.95 \%)$ & 3.12 \\
$\mathrm{HfS}_{2}(1 \mathrm{~T})$ & 3.65 & $5.74(-0.35 \%)$ & $11.47(-0.26 \%)$ & 5.72 \\
$\mathrm{HfSe}_{2}(1 \mathrm{~T})$ & 3.79 & $6.13(-0.33 \%)$ & $12.24(-0.16 \%)$ & 6.11 \\
$\mathrm{ZrS}_{2}(1 \mathrm{~T})$ & 3.70 & $5.74(-0.52 \%)$ & $11.46(-0.35 \%)$ & 5.71 \\
\hline $\mathrm{Str}$ & &
\end{tabular}

Structural parameters for monolayer (1L), bilayer (2L), and bulk $2 \mathrm{D}$ materials. $a$ represents the lattice constant, $t$ represents the thickness of monolayer and bilayer and $d_{i}$ denotes the interlayer distance in bulk. The numbers in parentheses represent the reduction in the interlayer distance from monolayer and bilayer to bulk

vacuum, is determined using optical measurements. ${ }^{20}$ This conclusion can be extended to other classes of $2 \mathrm{D}$ materials as well since Eq. (1) and Eq. (2) are material independent.

For the TMD results depicted in Figs. 2-3, we observe that the dielectric constant of the TMDs increases with the atomic number of the chalcogen, i.e., $\epsilon_{\mathrm{MTe}_{2}}>\epsilon_{\mathrm{MSe}_{2}}>\epsilon_{\mathrm{MS}_{2}}$. The out-of-plane dielectric response of $1 \mathrm{~T}$ TMDs is nearly independent of the number of layers. Mo-based TMDs show an increase of $13 \%$ in the out-ofplane dielectric constant when going from monolayer to bulk while their W-based TMDs show an increase of about $5 \%$. The larger increase in Mo-based TMDs overall is due to a higher interlayer coupling compared to the W-based TMDs. In line with our findings for h-BN, in the in-plane direction, 2H TMDs show $<10 \%$ variation with thickness for both the static and optical dielectric constant values. This negligible change in the in-plane dielectric constant from monolayer to bulk, compared to the outof-plane dielectric constant, is due to the atomic displacements in the plane in response to external fields. These in-plane displacements are practically unaltered by the interaction between the multiple layers.

Figures 1-3 also show that the electronic part dominates both the out-of-plane and in-plane dielectric constants for h-BN and TMDs with the $2 \mathrm{H}$ polytype structure. This is in line with what is found in most conventional semiconductors. ${ }^{28}$ The TMDs with the $1 \mathrm{~T}$ structure show a different behavior in the in-plane direction, however. In this direction, the ionic contribution is dominant for TMDs in the 1T structure, increasing to about 4.5 times its electronic counterpart. The marked difference between the ionic contribution in the $2 \mathrm{H}$ and the $1 \mathrm{~T}$ materials can be attributed to the breaking of both the rotational and the inversion symmetry for $1 \mathrm{~T}$ structures upon application of the in-plane field, unlike $2 \mathrm{H}$ structures where only the rotational symmetry is broken in response to an in-plane field. The breaking of this additional symmetry leads to an additional dipole which causes a large ionic response to external fields. To validate that the main difference is the crystal structure and not the atomic constituents, we calculated the ionic dielectric response for a 1T material, in particular $\left(\mathrm{HfS}_{2}\right)$ in a $2 \mathrm{H}$ structure. Indeed, we find that the ionic response in the in-plane direction reduces from 53.7 to 14.70 when we theoretically convert monolayer $\mathrm{HfS}_{2}$ from the $1 \mathrm{~T}$ to the $2 \mathrm{H}$ structure.

The finding that 1T TMDs have such a marked ionic contribution to their in-plane dielectric constant is detrimental for their perspective toward electronic transport. The difference between $\epsilon_{0}$ and $\epsilon_{\infty}$ determines the strength of the polar-optical (Fröhlich) interaction where a larger difference gives rise to larger polaroptical scattering rates, depressing mobility. Furthermore, the lack of mirror symmetry in the $1 \mathrm{~T}$ structure, giving rise to the very large in-plane ionic contribution, was also recently found to be detrimental for electronic transport because of strong acoustic phonon scattering with flexural mode. ${ }^{12}$ Both findings combined present a bleak outlook for TMDs in the 1T phase toward obtaining favorable electronic transport properties.

In conclusion, we have identified several patterns in the dielectric response of $\mathrm{h}-\mathrm{BN}$ and semiconducting TMDs that facilitate the selection, design, and modeling of $2 \mathrm{D}$ materials for use in future electronic devices. For h-BN, the in-plane component of the dielectric response is larger than its out-of-plane counterpart and is independent of the number of layers. On the other hand, the out-of-plane dielectric constant of h-BN shows an increase of about $15 \%$ from monolayer to bulk. For TMDs, we find that higher atomic number chalcogens give rise to a higher dielectric constant, and overall, the in-plane dielectric constant of TMDs is larger than its out-of-plane counterpart. The in-plane dielectric constant of TMDs, as in the case of h-BN, shows an insignificant variation with the number of layers in contrast to the out-of-plane response. Our calculations show that the electronic component dominates the overall dielectric response for all the 2D materials, except for TMDs with the 1T polytype structure. For the 1T TMDs in the in-plane direction, the static dielectric constant is, on average, about 4.5 times the dielectric constant at optical frequencies. This large difference between ionic and electronic dielectric response will give rise to large polar-optical phonon scattering rate and indicates a suppressed mobility in 1T TMDs.

\section{METHODS}

Details of the ab initio calculations

We calculate the structural and electronic properties of 2D materials using density functional theory (DFT) as implemented in the Vienna Ab Initio Simulation Package (VASP). ${ }^{29,30}$ The electron exchange correlation is treated in the Generalized Gradient Approximation (GGA) as proposed by Perdew-Burke-Ernzerhof (PBE). ${ }^{31}$ The cutoff energy is increased to twice the default energy cutoff, and the convergence criterion for electronic selfconsistent calculations is set to $10^{-8} \mathrm{eV}$. Setting the convergence criterion this strict is required to ensure an accurate determination of the dielectric properties. Grimme's van der Waals functional is used to correctly describe the interlayer distances. ${ }^{32}$ Brillouin zone integration is performed on a Monkhorst-Pack $n \times n \times 1 k$-space grid for free-standing layered structures and $n \times n \times n$ for bulk where $n$ is chosen so that all obtained values are converged. The value of $n$ which results in converged dielectric constants for bulk $2 \mathrm{H}$ TMDs, h-BN, and 1T TMDs turns out to be 12, 8 , and 16, respectively. We use cutoff energies of $520 \mathrm{eV}$ for S-based TMDs, $450 \mathrm{eV}$ for Se-based TMDs and $480 \mathrm{eV}$ for Te-based TMDs. The cutoff energy used for $\mathrm{h}-\mathrm{BN}$ is $400 \mathrm{eV}$. The length of the supercell used for different 2D materials

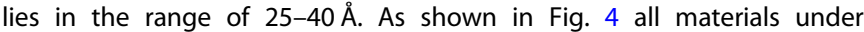
consideration have a hexagonal or tetragonal lattice with basis vectors $( \pm a \sqrt{3} / 2, a / 2, c)$, where $a$ is the in-plane lattice constant and $c$ is the dimension of the supercell for the few-layer materials and the out-of-plane lattice constant for the bulk materials. Each TMD unit cell has three atoms per layer, one metal $(\mathrm{M})$ and two chalcogens $(\mathrm{X})$, the $\mathrm{h}-\mathrm{BN}$ unit cells have one boron and one nitrogen atom per layer.

We consider both trigonal prismatic (2H) and octahedrally (1T) coordinated TMDs. Crystal structures with trigonal prismatic coordination are layered in an A-B stacking order; whereas, octahedrally coordinated 2D materials tend to stack in an A-A stacking order. ${ }^{33}$ Monolayer $\mathrm{h}-\mathrm{BN}$ is planar and adopts the honeycomb lattice structure. Multi-layered h-BN preferentially adopts the $A-A^{\prime}$ stacking order. ${ }^{34}$ The structural parameters of the $2 \mathrm{D}$ materials under consideration are determined by ionic relaxation in VASP and are listed in Table 3. 
$2 \mathrm{H}$

a.

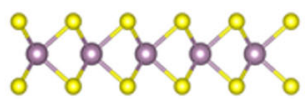

b.

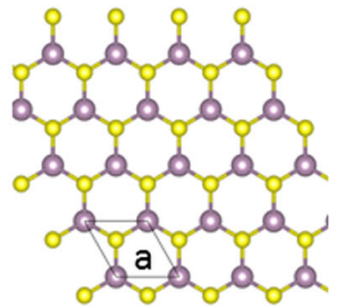

c.

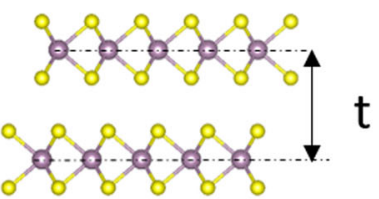

$1 T$
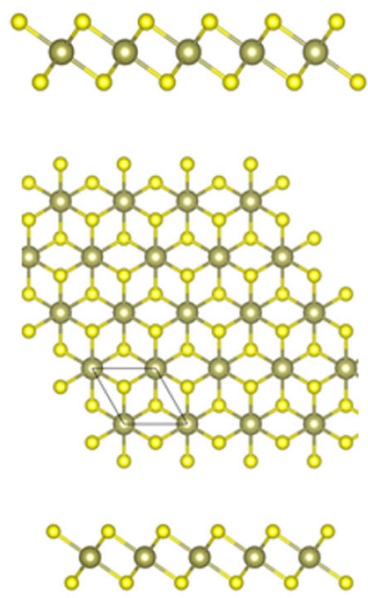

व $\sigma^{a} \sigma^{a} \sigma_{0}$
h-BN

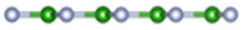

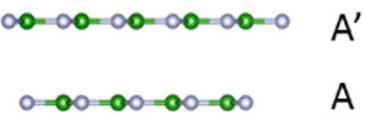

\section{Boron}

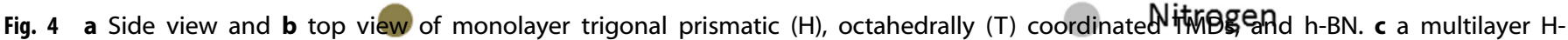
coordinated stack of TMDs in A-B configuration and a T-coordinated stack of TMDs in A-A configuration. Bilayer h-BN stacked in A-A' order. a is the in-plane lattice constant whereas $t$, the distance between the metal atom planes in the bilayer structure, corresponds to the monolayer thickness. These figures were generated using the Visualization for Electronic and Structural Analysis (VESTA) software package. ${ }^{39}$

Determining the electronic contribution to the in-plane and outof-plane dielectric constants

Monolayer and bilayer 2D materials are periodic in the $x-y$ plane; whereas, they are confined in the $z$-direction. Since, we use a DFT code that relies on a periodic plane wave basis, we employ the supercell approach. The non-physical interaction between periodic repetitions of layers is avoided by constructing a supercell where adjacent layers in the supercell are separated by a sufficiently large vacuum layer $(c \approx 10 a)$. We first calculate the macroscopic dielectric tensor $\epsilon_{\mathrm{SC}}$ of the supercell (SC) which contains the combined dielectric response of the 2D material and the vacuum. The dielectric tensor contains both the in-plane $\left(\epsilon_{\|}\right)$and outof-plane $\left(\epsilon_{\perp}\right)$ dielectric constants. Each of these values can be used to define the out-of-plane capacitance of the supercell as:

$$
C_{\mathrm{SC}_{\perp}}=\frac{\epsilon_{\mathrm{SC}_{\perp}}}{c} \text {. }
$$

In Eq. (3), $c$ is the length of the supercell. VASP calculates the dielectric tensor $\epsilon_{\mathrm{SC}}$ using the following equation:

$$
\epsilon_{a \beta}^{\mathrm{SC}}=\epsilon_{\mathrm{vac}}\left(\delta_{a \beta}+X_{a, \beta}\right) \text {. }
$$

In Eq. (4), $a, \beta \in\{x, y, z\}$ are the Cartesian directions, $\delta_{\alpha \beta}$ is the Kronecker delta, $\epsilon_{\alpha \beta}^{S C}$ is the dielectric tensor of the supercell, $\epsilon_{\mathrm{vac}}$ is the absolute dielectric permittivity with value of $8.85 \times 10^{-12} \frac{\mathrm{F}}{\mathrm{m}}$ and $X_{\alpha \beta}$ is the susceptibility tensor. The susceptibility tensor, $X_{a \beta}$, of the supercell is obtained using the density functional perturbation theory (DFPT) approach where the local field effects are included at the level of DFT as described in ref. ${ }^{35}$. The macroscopic values correspond to the dielectric response at optical frequency $(\omega \rightarrow \infty)$ where only the electrons respond to the external field and the ions remain frozen in their positions. Since, we are only interested in the macroscopic dielectric properties, we do not compute the frequency-dependent dielectric functions. We extract both the macroscopic out-of-plane $\left(\epsilon_{\mathrm{zz}}^{\mathrm{SC}}=\epsilon_{\mathrm{SC}_{\perp}}\right)$ and in-plane $\left(\epsilon_{\mathrm{xx}}^{\mathrm{SC}}, \epsilon_{\mathrm{yy}}^{\mathrm{SC}}\right)$ dielectric constant from the $\epsilon_{\alpha \beta}^{\mathrm{SC}}$ tensor. Since, we only consider hexagonal and tetragonal lattices, $\epsilon_{\mathrm{xx}}^{\mathrm{S} \beta}=\epsilon_{\mathrm{yy}}^{\mathrm{SC}}=\epsilon_{\mathrm{SC}}$ and $\epsilon_{\mathrm{xy}}^{\mathrm{SC}}=\epsilon_{\mathrm{xz}}^{\mathrm{SC}}=\epsilon_{\mathrm{yz}}^{\mathrm{SC}}=0$.

\section{Ionic contribution to the dielectric response}

The dielectric constant calculated using the method in the previous section represents only the electronic contribution, which neglects the response of the ions to the applied electric field. To account for the ionic response, we also determine the relaxed-ion dielectric tensor $\epsilon_{\alpha \beta}$ in a way similar to the way the clamped-ion dielectric tensor (Eq. (4)) was determined. In the case of the relaxed-ion dielectric tensor, the susceptibility tensor is modified to take into account the atomic displacements as given by Eq. (5). ${ }^{35}$

$X_{a \beta}=\tilde{X}_{a \beta}+\Omega_{0}^{-1} Z_{m a}\left(K^{-1}\right)_{m n} Z_{n \beta}$.

In the above equation $\tilde{X}_{\alpha \beta}$ represents the dielectric susceptibility which is purely electronic, $K_{m n}$ is the force-constant matrix and $Z$ is the Born dynamical effective charge tensor. ${ }^{35}$ Here, $\Omega_{0}$ represents the unit cell volume and $m, n$ represent a composite index capturing both the atom under consideration and the displacement direction. ${ }^{35}$

\section{Eliminating the contribution of the vacuum in the dielectric response}

The dielectric constant $\left(\epsilon_{\mathrm{SC}}\right)$ values obtained in the previous section represent the combined dielectric constant of the $2 \mathrm{D}$ material surrounded by a large vacuum. To distill the dielectric constant of the 2D material, we eliminate the contribution of the vacuum by invoking the principle of equivalent capacitance. For the out-of-plane direction, the capacitance of the supercell $C_{\mathrm{SC}}$ is the series combination of the vacuum capacitance $C_{\mathrm{vac}}$ and the $2 \mathrm{D}$ material capacitance $C_{2 \mathrm{D}}$,

$$
C_{\mathrm{SC}}^{-1}=C_{\mathrm{vac}}^{-1}+C_{2 \mathrm{D}}^{-1} \text {, }
$$

or, in terms of the dielectric constants:

$$
\frac{c}{\epsilon_{\mathrm{SC}, \perp}}=\frac{c-t}{\epsilon_{\mathrm{vac}}}+\frac{t}{\epsilon_{2 \mathrm{D}, \perp}},
$$

where $\epsilon_{2 \mathrm{D}}$ is the dielectric constant corresponding to the 2D material with thickness $t$. Eq. (7) yields the dielectric constant of the 2D material as outlined in Eq. (1). Similarly for the in-plane direction, the equivalent capacitance can be written as a parallel combination of the 2D material and the vacuum capacitance, yielding the in-plane dielectric constant of the 2D material as mentioned in Eq. (2). Both Eq. (1) and Eq. (2) can also be alternatively obtained from the effective medium approach, which is a microscopic view of the dielectric response as explained by Aspnes in ref. ${ }^{36}$. The linear dependence on supercell size (interlayer separation) was previously observed in Fig. 6 of ref. ${ }^{20}$. Because of the dependence of Eq. (1) and (2) on the thickness $t$, the extracted dielectric constants $\epsilon_{2 \mathrm{D}, \perp / \|}$ of the $2 \mathrm{D}$ materials, especially $\epsilon_{2 \mathrm{D}, \perp}$, are very sensitive to the value of 
thickness that is chosen as is shown in Supplementary Information. A judicious choice of the employed thickness of $2 \mathrm{D}$ materials under study is thus essential.

Previous work by Kumar et al. (ref. ${ }^{26}$ ) studied the layer-dependence of the h-BN dielectric constant. The definition of thickness employed in ref. ${ }^{26}$ is the total extent of the polarization, where the cutoff is made at the points where the polarization reaches $<1 \%$ of its peak value. However, this choice is arbitrary and different results would be obtained if a $0.5 \%$ or a $5 \%$ cutoff would have been used, or, if the extent of the charge density would have been used rather than the extent of the polarization. Most importantly, this thickness, obtained from the polarization, is strongly different from the physical inter-layer distance, which is ultimately the most relevant thickness quantity when considering stacked 2D materials.

Instead, we define the thickness based on the van der Waals bond length analogous to the atomic radius. ${ }^{37}$ More specifically, we relax an $n+1$ layer structure, accounting for van der Waals interactions, and then determine the distance between the center of the bottom and the top layer respectively. This distance is then used as the thickness of the $n$-layer stack. We note that the dielectric constants we calculate can easily be modified for different thickness values by rescaling the dielectric constant to a new value using Eq. (1) and Eq. (2). The thickness of the 2D materials used for the work in this paper are listed in Table 3. Finally, it is worth noting that the in-plane dielectric constant obtained for monolayer $\mathrm{MoS}_{2}$, rescaled using Eq. (2), is in close agreement to that reported in ref. ${ }^{38}$.

Data availability

The data that support the findings of this study is available at https://doi. org/10.17172/NOMAD/2020.07.17-1.

\section{ACKNOWLEDGEMENTS}

This material is based upon work supported by the National Science Foundation under Grant No. 1710066. We also acknowledge the support of Nanoelectronics Research Initiative's (NRI's) southwest Academy of Nanoelectronics (SWAN). We thank Prof. M. V. Fischetti, Prof. Christoper Hinkle and Prof. Robert Wallace for insightful discussions.

\section{AUTHOR CONTRIBUTIONS}

W.G.V. conceived the project. A.L. performed the simulations and the results obtained were analyzed by A.L., M.L.V.de.P. and W.G.V. A.L. wrote the paper with all the authors contributing to the discussion and preparation of the manuscript.

\section{ADDITIONAL INFORMATION}

Supplementary information accompanies the paper on the npj $2 D$ Materials and Applications website (https://doi.org/10.1038/s41699-018-0050-x).

Competing interests: The authors declare no competing interests.

Publisher's note Springer Nature remains neutral with regard to jurisdictional claims in published maps and institutional affiliations.

\section{REFERENCES}

1. Novoselov, K. et al. Electronic properties of graphene. Phys. Status Solidi (b) 244 4106-4111 (2007).

2. Butler, S. Z. et al. Progress, challenges, and opportunities in two-dimensional materials beyond graphene. ACS Nano 7, 2898-2926 (2013).

3. Ma, R. \& Sasaki, T. Two-dimensional oxide and hydroxide nanosheets: controllable high-quality exfoliation, molecular assembly, and exploration of functionality. Acc. Chem. Res. 48, 136-143 (2014).

4. Zhao, J. et al. Rise of silicene: a competitive 2D material. Prog. Mater. Sci. 83 24-151 (2016).

5. Liu, H. et al. Phosphorene: an unexplored 2D semiconductor with a high hole mobility. ACS Nano 8, 4033-4041 (2014).

6. Wang, Q. H., Kalantar-Zadeh, K., Kis, A., Coleman, J. N. \& Strano, M. S. Electronics and optoelectronics of two-dimensional transition metal dichalcogenides. Nat. Nano 7, 699-712 (2012)

7. Qian, X., Liu, J., Fu, L. \& Li, J. Quantum spin Hall effect in two-dimensional transition metal dichalcogenides. Science 346, 1344-1347 (2014).
8. Wilson, J. \& Yoffe, A. The transition metal dichalcogenides discussion and interpretation of the observed optical, electrical and structural properties. Adv. Phys. 18, 193-335 (1969).

9. Kang, M. et al. Universal mechanism of band-gap engineering in transition-metal dichalcogenides. Nano Lett. 17, 1610-1615 (2017).

10. Recent development of two-dimensional transition metal dichalcogenides and their applications. Mater. Today 20, 116-130 (2017).

11. Jariwala, D., Sangwan, V. K., Lauhon, L. J., Marks, T. J. \& Hersam, M. C. Emerging device applications for semiconducting two-dimensional transition metal dichalcogenides. ACS nano 8, 1102-1120 (2014).

12. Fischetti, M. V. \& Vandenberghe, W. G. Mermin-wagner theorem, flexural modes, and degraded carrier mobility in two-dimensional crystals with broken horizontal mirror symmetry. Phys. Rev. B 93, 155413 (2016).

13. Lopez-Sanchez, O., Lembke, D., Kayci, M., Radenovic, A. \& Kis, A. Ultrasensitive photodetectors based on monolayer mos2. Nat. Nanotechnol. 8, 497-501 (2013).

14. Negreira, A. S., Vandenberghe, W. G. \& Fischetti, M. V. Ab initio study of the electronic properties and thermodynamic stability of supported and functionalized two-dimensional sn films. Phys. Rev. B 91, 245103 (2015).

15. Balandin, A. A. et al. Superior thermal conductivity of single-layer graphene. Nano Lett. 8, 902-907 (2008)

16. Watanabe, K., Taniguchi, T. \& Kanda, H. Direct-bandgap properties and evidence for ultraviolet lasing of hexagonal boron nitride single crystal. Nat. Mater. 3, 404 (2004).

17. Chan, M. Y. et al. Suppression of thermally activated carrier transport in atomically thin mos 2 on crystalline hexagonal boron nitride substrates. Nanoscale $\mathbf{5}$, 9572-9576 (2013).

18. Withers, F., Bointon, T. H., Hudson, D. C., Craciun, M. F. \& Russo, S. Electron transport of ws 2 transistors in a hexagonal boron nitride dielectric environment. Sci. Rep. 4 (2014).

19. Das, S., Gulotty, R., Sumant, A. V. \& Roelofs, A. All two-dimensional, flexible, transparent, and thinnest thin film transistor. Nano Lett. 14, 2861-2866 (2014).

20. Hüser, F., Olsen, T. \& Thygesen, K. S. How dielectric screening in two-dimensional crystals affects the convergence of excited-state calculations: monolayer mos 2 . Phys. Rev. B 88, 245309 (2013).

21. Latini, S., Olsen, T. \& Thygesen, K. S. Excitons in van der waals heterostructures: the important role of dielectric screening. Phys. Rev. B 92, 245123 (2015).

22. Andersen, K., Latini, S. \& Thygesen, K. S. Dielectric genome of van der waals heterostructures. Nano Lett. 15, 4616-4621 (2015).

23. Yu, E. K., Stewart, D. A. \& Tiwari, S. Ab initio. Phys. Rev. B 77, 195406 (2008).

24. Bao, W., Cai, X., Kim, D., Sridhara, K. \& Fuhrer, M. S. High mobility ambipolar mos2 field-effect transistors: substrate and dielectric effects. Appl. Phys. Lett. 102, 042104 (2013).

25. March, N. \& Tosi, M. Polymers, Liquid Crystals, and Low-dimensional Solids. (Plenum Press, New York, USA, 1984).

26. Kumar, P., Chauhan, Y. S., Agarwal, A. \& Bhowmick, S. Thickness and stacking dependent polarizability and dielectric constant of graphene-hexagonal boron nitride composite stacks. J. Phys. Chem. C 120, 17620-17626 (2016).

27. Santos, E. J. \& Kaxiras, E. Electrically driven tuning of the dielectric constant in mos2 layers. ACS Nano 7, 10741-10746 (2013).

28. Madelung, O. Semiconductors-basic data. (Springer Science and Business Media, Berlin, Germany, 2004).

29. Kresse, G. \& Furthmüller, J. Efficient iterative schemes for ab initio total-energy calculations using a plane-wave basis set. Phys. Rev. B 54, 11169-11186 (1996).

30. Blöchl, P. E. Projector augmented-wave method. Phys. Rev. B 50, 17953-17979 (1994)

31. Perdew, J. P., Burke, K. \& Ernzerhof, M. Generalized gradient approximation made simple. Phys. Rev. Lett. 77, 3865-3868 (1996).

32. Grimme, S. Semiempirical gga-type density functional constructed with a longrange dispersion correction. J. Comput. Chem. 27, 1787-1799 (2006).

33. Wu, R. J., Odlyzko, M. L. \& Mkhoyan, K. A. Determining the thickness of atomically thin mos 2 and ws 2 in the tem. Ultramicroscopy 147, 8-20 (2014).

34. Constantinescu, G., Kuc, A. \& Heine, T. Stacking in bulk and bilayer hexagonal boron nitride. Phys. Rev. Lett. 111, 036104 (2013).

35. Wu, X., Vanderbilt, D. \& Hamann, D. Systematic treatment of displacements, strains, and electric fields in density-functional perturbation theory. Phys. Rev. $B$ 72, 035105 (2005).

36. Aspnes, D. Local-field effects and effective-medium theory: a microscopic perspective. Am. J. Phys. 50, 704-709 (1982).

37. Pauling, L. The nature of the chemical bond and the structure of molecules and crystals: an introduction to modern structural chemistry, vol. 18 (Cornell University Press, USA, 1960).

38. Sohier, T., Calandra, M. \& Mauri, F. Two-dimensional fröhlich interaction in transition-metal dichalcogenide monolayers: theoretical modeling and firstprinciples calculations. Phys. Rev. B 94, 085415 (2016). 
39. Momma, K. \& Izumi, F. VESTA3 for three-dimensional visualization of crystal, volumetric and morphology data. J. Appl. Crystallogr. 44, 1272-1276 (2011).

cC) (i) Open Access This article is licensed under a Creative Commons Attribution 4.0 International License, which permits use, sharing, adaptation, distribution and reproduction in any medium or format, as long as you give appropriate credit to the original author(s) and the source, provide a link to the Creative Commons license, and indicate if changes were made. The images or other third party material in this article are included in the article's Creative Commons license, unless indicated otherwise in a credit line to the material. If material is not included in the article's Creative Commons license and your intended use is not permitted by statutory regulation or exceeds the permitted use, you will need to obtain permission directly from the copyright holder. To view a copy of this license, visit http://creativecommons. org/licenses/by/4.0/.

(c) The Author(s) 2018 